\title{
Failure of the vascular hypothesis of multiple sclerosis in a rat model of chronic cerebrospinal venous insufficiency
}

\author{
Maha M.A. Zakaria ${ }^{1}$, Shahira Y. Mikhael $^{1}$, Azza K. Abu Hussein ${ }^{1}$, Rania A. Salah El-din ${ }^{1}$, Hany W. Abd El-Malak ${ }^{1}$, \\ Iman H. Hewedi ${ }^{2}$, Hany S. Nadim ${ }^{1}$ \\ ${ }^{1}$ Department of Anatomy, Faculty of Medicine, Ain Shams University, Cairo, ${ }^{2}$ Department of Pathology, Faculty of Medicine, \\ Ain Shams University, Cairo, Egypt
}

\begin{abstract}
Chronic cerebrospinal venous insufficiency (CCSVI) is a series of stenotic malformations in the cerebrospinal venous outflow routes, which is postulated to cause multiple sclerosis (MS). The hypotheses assumed that CCSVI leads to iron deposition which triggers inflammation and demyelination in MS. Invasive endovascular treatment of CCSVI was initiated based on the previous theory. The present study was designed to validate this hypothesis using a rat model of CCSVI. Bilateral jugular vein ligation (JVL) was performed on female albino rats ( $n=15)$, and sham-operated rats $(n=15)$ were used as a control group. The rats were followed clinically for eight months and neurological examination detected no weakness or paralysis in the operated rats. At the end of the experiment, the rats were sacrificed and the brains were processed for histopathological examination of tissue sections stained by hematoxylin and eosin, myelin stain, silver impregnation, iron stain and immunohistochemical preparations for GFAP, CD68 and CD45. Semithin sections stained with toluidine blue were also examined. In the JVL group, increased iron deposition in the white matter was detected. An increase in the size and number of astrocytes along with increased GFAP immunoreactivity denoting reactive gliosis was also noted in the JVL group. However, no signs of demyelination, inflammation or axonopathy were detected. This study revealed that iron deposition in the JVL group as a model for CCSVI was not associated with cardinal histopathological findings of MS. It is therefore recommended that the invasive endovascular treatment of CCSVI should be reconsidered and further controlled clinical studies be carried out to provide a better understanding of the pathogeneses of MS.
\end{abstract}

Key words: CCSVI, multiple sclerosis, animal model, jugular veins, iron overload.

\section{Introduction}

Chronic cerebrospinal venous insufficiency (CCSVI) comprises a series of stenotic malformations affecting the extracranial cerebrospinal venous outflow routes, mainly the internal jugular and azygous veins [33]. The chronic insufficient venous drainage occur- ring in the course of CCSVI was postulated to cause multiple sclerosis (MS) through the deposition of iron in the walls of the congested veins and in the brain tissue [43]. Multiple sclerosis is a chronic incurable demyelinating disease of the central nervous system, which affects up to 2.5 million people worldwide [15]. 
Multiple sclerosis is described as focal inflammatory demyelination that leads to slowing or loss of impulse transmission [35]. The development of MS occurs in three stages: inflammation, demyelination and axonal damage [23].

Iron overload is hypothesized to act as a causal factor in the development of MS lesions [41], either directly via oxidative stress or indirectly by causing the primary activation of the autoimmune cascade [32].

The potential link between the pathogenesis of MS and CCSVI has generated tremendous interest in the scientific community and in the news media [17].

To date, a limited trial has been attempted to create an animal model of CCSVI, bilateral jugular venous ligation have been performed and the mice models have been evaluated for inflammation and demyelination. However, detection of iron and explaining its role in the pathological changes attributed to CCSVI was never attempted [3].

Given the insufficient evidence and the conflicting data concerning the role of CCSVI in the etiology of MS and the source of iron in the course of MS, the present study aims to clarify if CCSVI has any role in MS pathogenesis in a rat model.

\section{Material and methods}

\section{Animals and ethics statement}

After approval of the CARE (Committee of animal research ethics) of the Faculty of Medicine, Ain Shams University, thirty female adult six-month-old Wistar rats of an average weight of 160 gm were obtained from the animal house of the Medical Research Center (MRC) of the Faculty of Medicine, Ain Shams University and were randomly assigned to either a jug ular vein ligation group $(n=15)$ or a sham-operated group $(n=15)$. The animals were given a standard diet and tap water ad libitum, housed as three rats/cage and kept in a temperature-controlled room $\left(23 \pm 1^{\circ} \mathrm{C}\right)$ with a 12 : 12 hours light/dark cycle.

\section{Jugular veins ligation}

Under anesthesia using an intraperitoneal injection of ketamine $75 \mathrm{mg} / \mathrm{kg}$ and intramuscular chlorpromazine hydrochloride $20 \mathrm{mg} / \mathrm{kg}$ and complete aseptic conditions [45], a midline neck incision was performed taking care not to injure the rat salivary glands. The external jugular veins were exposed bilaterally and ligated in their upper portion just distal to its formation by the union of the anterior and posterior facial veins to avoid blockage of salivary glands drainage using sterile silk 4-0 sutures. The skin closure was done using absorbable vicryl sutures. For six successive postoperative days, wound cleaning was performed and Cefotaxime $40 \mathrm{mg} / \mathrm{kg}$ intramuscular injection was given twice daily.

A control group (sham-operated group) was exposed to the same operative procedures and postoperative precautions but without jugular veins ligation.

\section{Neurological examination}

The rats were followed up clinically using the standard 5-point "Experimental autoimmune encephalomyelitis" staging system according to Chen et al. [9], starting from one month post-surgery: 0 = no signs, 1 = complete tail limpness without limb weakness, 2 = limb weakness without obvious paralysis on ambulation, 3 = one limb with partial paralysis (voluntary movements are still possible), $4=$ one limb with complete paralysis, and $5=$ moribund.

Tail limpness was tested by fixation of the tail base and observing the tone of the tail, limb weakness was defined by an impaired righting reflex; the rats have difficulty turning over after being laid down on their back, complete paralysis was defined as a total loss of righting reflex.

\section{Obtaining brain tissue for assessment}

After eight months and under anesthesia using ketamine $75 \mathrm{mg} / \mathrm{kg}$ IP and chlorpromazine hydrochloride $20 \mathrm{mg} / \mathrm{kg}$ IM [45], perfusion fixation of the rat brain was performed; and thoracotomy was done and $4 \%$ warm formalin in phosphate buffer saline was infused in the rat's left ventricle [14]. Decapitation was done, then the skull was opened and the brain was extracted.

A coronal segment of the brain was cut between a level of $8 \mathrm{~mm}$ to $15.3 \mathrm{~mm}$ from the beginning of the olfactory bulb which is equivalent to bregma $1 \mathrm{~mm}$ to bregma $-6.3 \mathrm{~mm}$ according to stereotaxic coordinates by Paxinos and Watson [30]. The selected segment comprises most of the brain white matter bundles. The brain segment was immersed in 10\% formalin in phosphate buffer saline for processing of paraffin blocks.

For preparation of semithin sections, samples of white matter $\left(1 \mathrm{~mm}^{3}\right)$ from various areas were har- 
vested and fixed in a solution of 3\% phosphate buffered glutaraldehyde ( $\mathrm{pH} 7.3)$ overnight at $4^{\circ} \mathrm{C}$.

\section{Histopathological examination and histochemical staining}

6-8 $\mu \mathrm{m}$ sections were prepared from paraffin blocks for the following stains:

1. Hematoxylin and Eosin (H\&E) stain for routine histopathological assessment: the major white matter tracts observed in the coronal brain tissue sections were carefully examined.

2. Luxol fast blue-cresyl violet stain for myelin using the luxol fast blue-cresyl echt violet stain kit (American Master kit, USA) [42].

3. Silver axonal staining using modified Glees technique [5] and Bielschowsky's technique [11].

4. Perls Prussian blue stain for iron [5].

\section{Immunohistochemical staining}

Immunohistochemical staining was carried out on $4 \mu \mathrm{m}$ sections of formalin-fixed paraffin embedded tissue using Dako autostainer with standard labeled streptavidin- biotin-peroxidase complex technique and diaminobenzidine (DAB) served as the chromogen. Staining was performed applying a polyclonal rabbit antibody against glial fibrillary protein (GFAP) (Dako, Code IS524, ready-to-use), monoclonal mouse antibody against CD68 (Dako, Code IS609, Clone KP1, ready-to-use), monoclonal mouse antibody against CD45 (Dako, Code IS751, Clones 2B11 + PD7/26, readyto-use). Rat brain, rat tonsil and rat spleen tissues were used as positive control for GFAP, CD68, CD45 immunostains, respectively. Negative control was achieved by performing the staining procedures with omission of the primary antibody.

\section{Toluidine blue staining of semithin sections from resin-embedded blocks}

The samples were washed off the fixative preparation in phosphate buffer for a period of 4 hours, then post fixed in $1 \%$ buffered osmium tetroxide for $1-2$ hours at $4^{\circ} \mathrm{C}$. The tissue pieces were subsequently washed twice in phosphate buffer and then dehydrated in ascending grades of ethanol. Clearing was done in propylene oxide for 20 minutes at room temperature. Infiltration was then done by using equal parts of propylene oxide and Epon 812 for overnight. The specimens were embedded in gelatin capsules filled with fresh Epon. The capsules were placed in the oven at $60^{\circ} \mathrm{C}$ for 48 hours to allow polymerization. Finally, semithin sections were cut at one-micron thickness with a glass knife and were stained with $1 \%$ toluidine blue stain dissolved in $1 \%$ borax for approximately $30-60$ seconds at $60-70^{\circ} \mathrm{C}$.

\section{Computer image analysis}

"TS View" computer image analysis software version 6.2.4.5 was used to quantitate the number of myelinated fibers and the number of astrocytes per microscopic field as well as the surface area of the astrocytic cell.

$\mathrm{NIH}$ "Image J" computer image analysis software version $1.40 \mathrm{~g}$ was used to measure the area of iron deposits, area of stained myelin as well as area stained immunohistochemically by GFAP per microscopic field.

For each of the previous entries, measurements were taken from six microscopic fields per slide, six slides per rat and six rats per group.

Counting the myelinated fibers in semithin sections per microscopic field was done using the 100X objective lens. The number of astrocytes per microscopic field was counted using the $40 \times$ objective lens. The surface area of astrocytes in square micrometer was measured using the 100x objective lens ( 6 astrocytes were measured for each case and the mean surface area of the individual astrocyte was then calculated). For measurement of area percentage of iron and GFAP per microscopic field, the $4 \times$ objective lens was used.

Calibration of the software was done for each microscopic magnification in order to translate pixels into micrometers. This was done with the aid of a stage micrometer.

For measurement of area percentage per microscopic field used for quantitation of iron and GFAP, images were split into RGB stacks, then the red stack was chosen and adjusted to grey scale threshold to mark the stained areas-areas of positive immunoreactivity with a red-colored binary mask. Then, the percentage of these areas in relation to the microscopic field was subsequently calculated.

\section{Statistical analysis}

SPSS software (Version 13.0) was used. Comparison of means between the control and experimental groups was done using independent-samples t-test with $p$ value of $<0.05$ was considered significant and $<0.01$ was considered highly significant. 


\section{Results}

\section{Chronic cerebrospinal venous insufficiency in rats does not result in neurological deficit}

Clinical follow-up of the rats performed using the standard 5-point "Experimental autoimmune encephalomyelitis" staging system revealed no neurological signs in both the sham-operated control group and the jugular vein ligation group. Monthly follow-up started one month post-surgery revealed preserved tone and movement of the rat tail on fixation of the base of the tail with no paralysis or flaccidity. Normal righting reflex was observed.

\section{Histopathological examination and histochemical staining results}

Examination of white matter tracts at the level of bregma $1 \mathrm{~mm}$, bregma $-1.4 \mathrm{~mm}$, and bregma $-2.8 \mathrm{~mm}$ as they appeared craniocaudally and dorsoventrally included the cingulum, the corpus callosum, the external capsule, the discrete patches of fibers dispersed in the caudate-putamen, the anterior commissure, the medial forebrain bundle, the lateral olfactory tract, the ventral hippocampal commissure, the dorsal hippocampal commissure, the stria medullaris thalami, the fimbria, the stria terminalis, the internal capsule, the fornix, the mammillothalamic tract, and the optic tract. The following were also examined: the white matter structures apparent at the level of the superior colliculus of the midbrain corresponding to bregma $-6.3 \mathrm{~mm}$ comprised of the superior colliculus, the medial longitudinal fasciculus, cerebral peduncle, the medial lemniscus, and the alveus.

\section{Histopathological examination by $H \& E$}

The examined white matter tracts in both jugular vein ligation group and sham-operated control group exhibited normal appearing white matter with no detectable hypodense patches suggesting demyelination. No signs of congestion or inflammatory cell infiltration were noted in both groups. Mild widening of perivascular Virchow Robin space with surrounding mild brain tissue edema was observed in the jugular vein ligation group compared to the sham-operated control group.

\section{Myelin luxol fast blue/cresyl violet stain}

The white matter showed reserved integrity with no foci of demyelination on light microscopic exam- ination of Luxol fast blue/cresyl violet stained rat brain coronal sections in both the sham-operated control group and jugular vein ligation group (Fig. 1A-B). Sharp discrimination between the white matter, which was stained deep blue, and grey matter, which acquired light blue color, was exhibited by the stain.

Computerized image analysis revealed that the percentage of areas stained for myelin per microscopic field in the sham-operated group and in the JVL group were $21.86 \% \pm 10.93 \%$ and $23.15 \% \pm 11.73 \%$, respectively. The difference between the two groups was statistically non-significant $(p=0.813)$ (Fig. 2).

\section{Silver stains}

Light microscopic examination of silver stained coronal sections of the rat brain in the JVL group revealed similar results to the control group. No alteration in the organization of the major white matter structures with no apparent tangles or swellings in the fibers were noted with Bielschowsky stain and modified Glees technique (Fig. 1C-D).

\section{Iron Perls Prussian blue stain}

Light microscopic examination of Perls Prussian blue stained coronal sections of the brain of the rats in the sham-operated control group showed minimal or negative iron deposits (Fig. 1E). On higher magnification, this trivial iron deposition was observed within the oligodendrocytes, the endothelial lining of the blood vessels as well as in the surrounding brain tissue.

Regarding the jugular vein ligation group, markedly increased iron deposition was noted (Fig. 1F). The iron deposits were aggregated mainly in white matter tracts including the corpus callosum, the cingulum, and the external capsule, the white matter tracts within the caudate-putamen complex, and the anterior commissure. Moderate iron deposits were seen in the fimbria and the internal capsule and the stria terminalis, minimal deposits were observed in the optic tract, the fornix, the mammillothalamic tract and the medial longitudinal bundle. On higher magnification, the iron deposits were observed within the oligodendrocytes as well as intercellular deposits. The blood vessels captured on higher magnification photomicrographs showed extensive iron deposits in the endothelial lining of the blood vessels as well as a marked increase in iron in the surrounding brain tissue. 

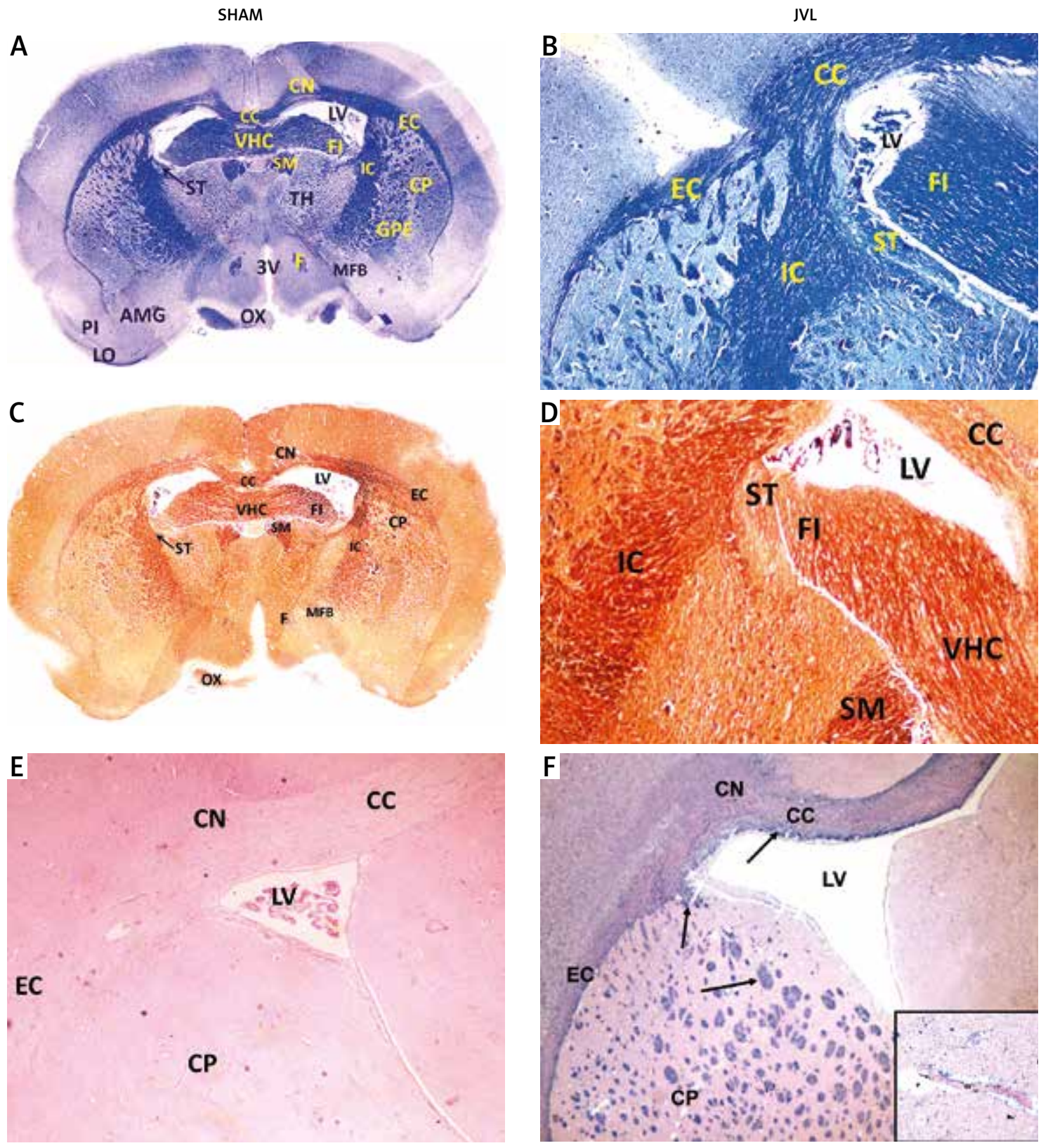

Fig. 1. Reserved white matter integrity with no foci of demyelination in both sham-operated control group (A: reconstructed picture) and JVL group (B); Luxol fast blue/cresyl violet stain. No alteration in the organization of the major white matter structures with no apparent tangles or swellings in the fibers in both sham-operated control group (C: reconstructed picture) and JVL group (D); Bielschowsky silver stain. No apparent iron deposit in sham-operated control group (E), while a marked increase in iron deposition is noted in the JVL group, mainly noted in white matter tracts (arrows) (F), inset shows iron deposits in endothelial cells (arrows) of a blood vessel "BV" and intercellular deposit in surrounding brain tissue (arrowheads) with mild widening of the perivascular space "P"; Perls Prussian Blue stain. Site of sections at coronal levels corresponding to bregma $-1.4 \mathrm{~mm}$ for $\mathrm{A}, \mathrm{B}, \mathrm{C}$, and $\mathrm{D}$ and bregma $-1.2 \mathrm{~mm}$ for $\mathrm{E}$ and $\mathrm{F}$; original magnification for all $\times 40$ and $\times 1000$ for inset. Abbreviations: amygdaloid complex $(A M G)$, corpus callosum $(C C)$, cingulum $(C N)$, caudate-putamen (CP), external capsule (EC), fimbria (FI), fornix (F), internal capsule (IC), globus pallidus externa (GPE), lateral olfactory tract (LO), lateral ventricle (LV), medial forebrain bundle (MFB), optic chiasma (OX), piriform cortex (PI), stria medullaris thalamai (SM), stria terminalis (ST), thalamus $(\mathrm{TH})$, ventral hippocampal commissure (VHC), third ventricle (3V). 


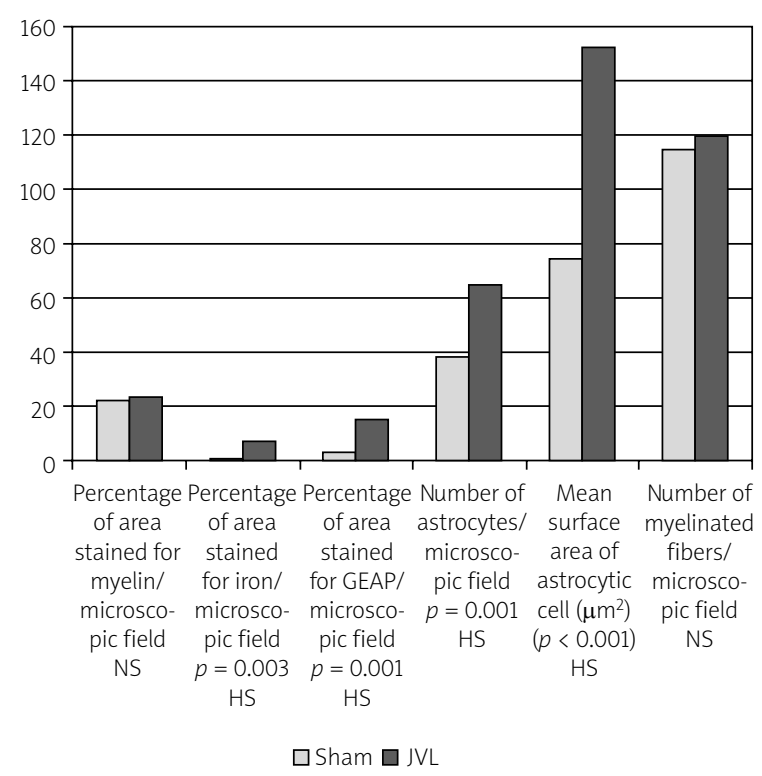

Fig. 2. Myelin content, iron content, GFAP immunohistochemical results, and number of myelinated fibers in the sham-operated group and JVL group as assessed by computerized image analysis; NS - non-significant; HS - highly significant.

Upon computer image analysis, the percentage of areas stained for iron per microscopic field in the sham-operated group was $0.93 \% \pm 1.37 \%$ compared to $7 \% \pm 5.66 \%$ in the JVL group. This difference was statistically highly significant $(p=0.003)$ (Fig. 2).

\section{Immunohistochemical staining results}

\section{GFAP immunostaining reveal astrogliosis in rats with CCSVI}

The stain highlighted the astrocytic cells mainly in the perivascular localization with apparent astrogliosis observed in the JVL group compared to the sham-operated group. The reactive astrocytes were distributed rather evenly in the white tracts examined with a slight increase in numbers at the corpus callosum and external capsule. This astrogliosis is evidenced as a marked increase in size and number of reactive astrocytes in the JVL group compared to the sham-operated group which appeared as large cells with highly branching thick and intensely staining processes.

This astrogliosis observed by light microscopic examination of the GFAP stained section in the JVL group was further confirmed upon comparing the
$J V L$ group to the sham-operated group regarding the area percentage of GFAP immunoreactivity per microscopic field $(14.81 \pm 6.2$ vs. $3.21 \pm 0.55)$ and the number of astrocytes per microscopic field (64.67 \pm 8.62 vs. $37.83 \pm 7.94)$, as well as the mean surface area of the astrocyte in $\mu 2(152.88 \pm 61.44$ vs. $74.37 \pm$ 42.39) via computerized image analysis with a highly significant statistical difference, $p<0.001$ (Figs. 2 and 3).

\section{CD68 and CD45 immunostaining reveal lack of inflammatory response in rats with CCSVI}

Neither microglial/macrophage nor lymphocytes could be detected in either perivascular or brain-parenchymal localization in both sham-operated and JVL groups (Fig. 4).

\section{Results of semithin toluidine blue stained sections examination}

Light microscopic examination of toluidine blue stained semithin sections from the rat brain of both the sham-operated control group and the JVL group revealed similar findings with unremarkable myelinated nerve fibers, oligodendrocytes, and astrocytes (Fig. 5). Examination of nerve fibers in the corpus callosum and the caudate-putamen showed myelination of most of the fibers with the myelin appearing as a continuous dense cuff surrounding the axons with no apparent vacuolation or thinning. The axons appeared pale and homogenous (Fig. 5).

Computerized image analysis revealed no statistical significant difference between the number of myelinated fibers per microscopic field in the JVL group $(119.83 \pm 29.2)$ compared to the sham-operated group (114.5 \pm 32.24$), p=0.770$ (Fig. 2).

\section{Discussion}

This study was designed to validate the hypothesis that CCSVI leads to inflammation and demyelination in MS through the deposition of iron in the brain.

The present study showed that even though the rat model of CCSVI showed extensive iron deposition and reactive astrogliosis mostly in white matter, no signs of demyelination were detected. Also no signs of inflammation were observed as confirmed by negative immunoreactivity for both CD68 and CD45. The neurological follow-up of the JVL group and the sham-operated group showed similar results with no paralysis or weakness and intact righting reflex. 
SHAM
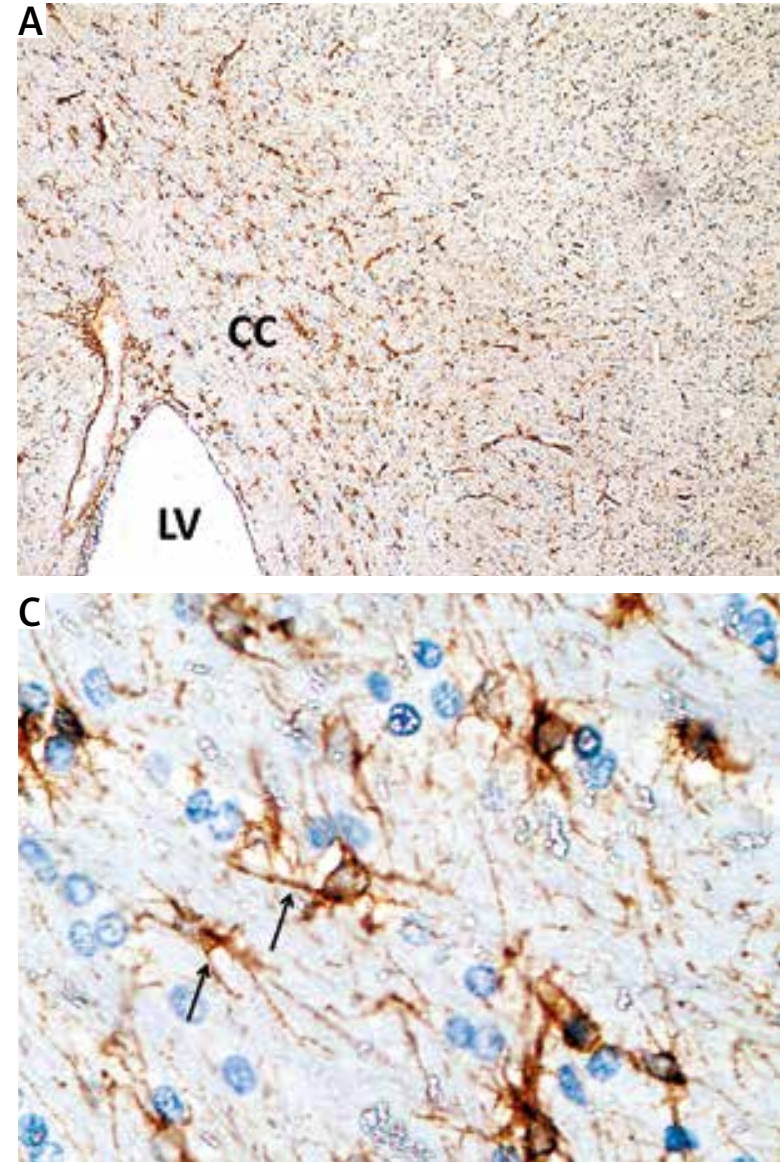

JVL
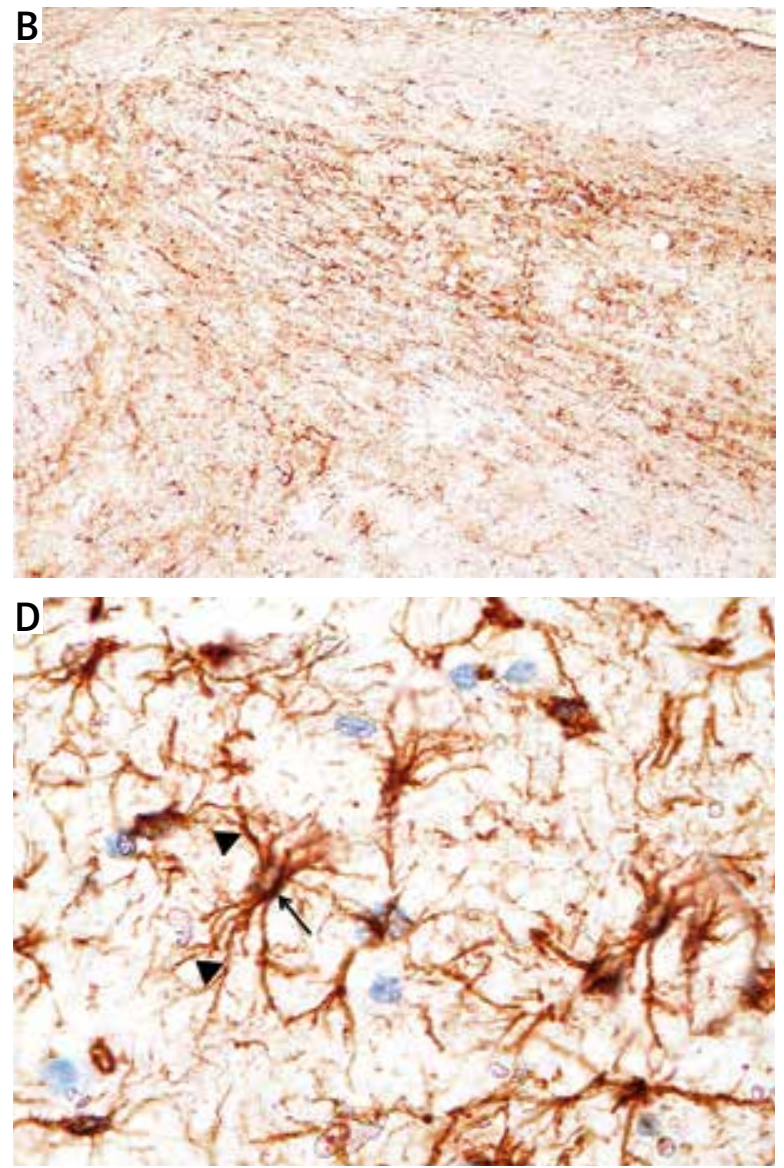

Fig. 3. GFAP immunostaining: apparent moderate positive staining of white matter in the sham-operated group (A); while intense staining was noted in corpus callosum (CC) adjacent to the lateral ventricle (LV) from the JVL group (B); original magnification $\times 100$. Reactive astrocytes in the sham-operated group (C) and JVL group (D) with apparent astrogliosis observed in the JVL group evidenced as a marked increase in size and number of astrocyte with highly branching thick and intensely staining processes; original magnification $\times 1000$.
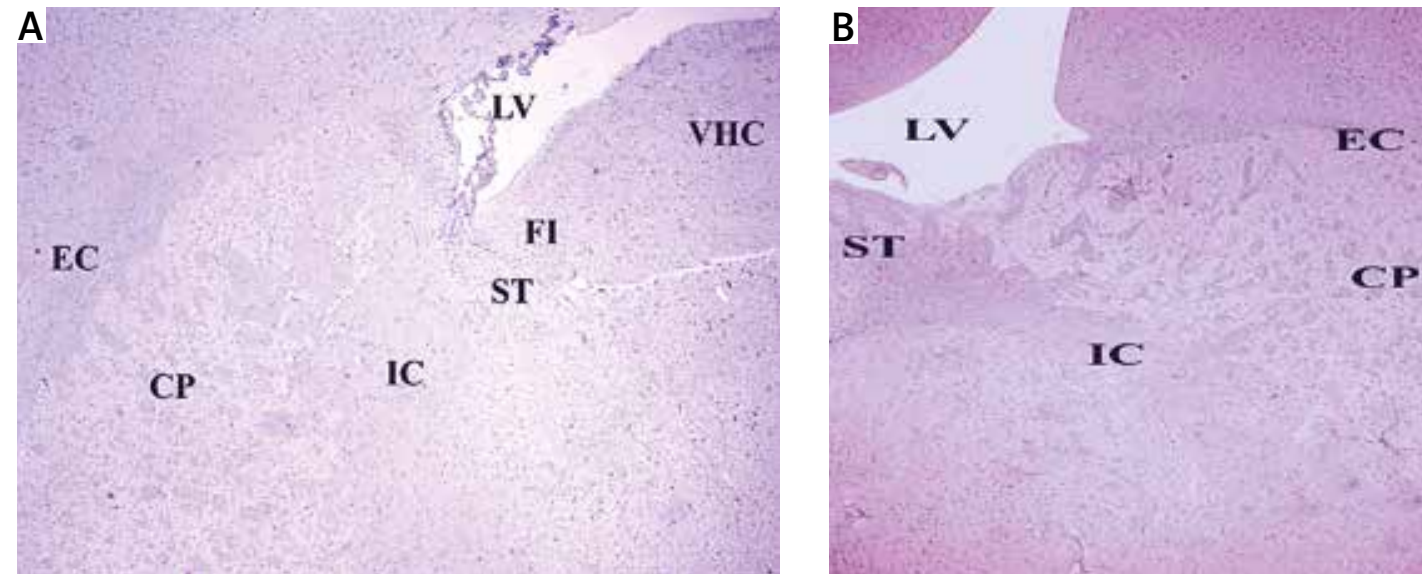

Fig. 4. No detectable inflammatory response with lack of microglial/macrophage (A) and lymphocytes (B) as assessed by CD68 and CD45 immunostaining, respectively, in the JVL group; the site of section at coronal levels corresponding to bregma -1.4 and $-2.8 \mathrm{~mm}$, respectively, original magnification $\times 40$. Abbreviation: caudate-putamen (CP), external capsule (EC), fimbria (FI), internal capsule (IC), stria terminalis (ST), lateral ventricle (LV), ventral hippocampal commissure (VHC). 


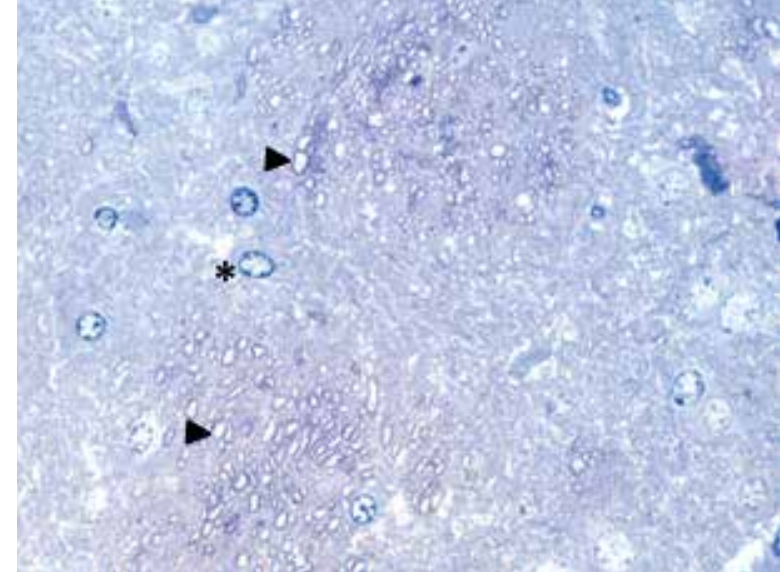

Fig. 5. Semithin section examination of nerve fibers in the caudate-putamen in the JVL group exhibited myelination of the nerve fibers (arrowheads) with no apparent vacuolation or thinning, astrocytes $\left(^{*}\right)$ are also observed; Toluidine blue stain. Original magnification $\times 1000$.

Jugular vein stenosis was found in some studies to exist in $91 \%$ of MS patients with $14 \%$ showing bilateral stenosis $[26,52]$. The cerebral venous outflow in the rat is carried mainly by the external jugular vein with contribution from the vertebral vein, unlike human circulation, the internal jugular vein is almost vestigial in the rat [4]. In the present study, total ligation of the external jugular vein bilaterally in the rat model was performed to maximize the effect on the cerebral venous circulation.

Bilateral jugular vein stenosis in CCSVI was associated with activation of the cervical and intracranial collateral circles shunting the blood towards the superior vena cava and the azygos venous system, respectively [48]. The activation of these collateral circuits was confirmed by $\mathrm{CT}$ venogram in the JVL animal model used by Atkinson et al. [3]; in the present study, the rat model was used replacing the original mouse model, the cerebral venous drainage in both species is similar as well as the brain structure with few minor differences $[29,44]$.

Mimicking the exact pattern of the venous malformations in CCSVI in a rat model was inaccessible due to the difficulty and high mortality encountered with ligation of the azygos or vertebral veins in rats.

The head position in humans differs from that of the rat which impacts the cerebral venous drainage. In humans the upright position places the internal jugular veins above the heart causing their collapse and shifts the cerebral venous drainage towards the vertebral vein and azygos system [46]. As rats are quadrupeds, the jugular veins are less liable to compression and play a major role in the cerebral venous outflow [29], which accounts for the use of the jugular veins in the present study.

Although the study of Atkinson et al. [3], which employed mice as an animal model of CCSVI, extended over a period of six months, the present experiment extended over a period of eight months in rats. This period covers almost one third of the life span of albino rats [38]. In addition, this time period should be sufficient for the occurrence of pathological manifestations of MS i.e. inflammation, demyelination and axonal injury. Taking into consideration the occurrence of MS in humans in their third or fourth decade [27] and that in the present experiment JVL was performed bilaterally and the venous occlusion was complete, and this would enhance the occurrence of such manifestations if the hypothesis of Zamboni et al. [51] was valid.

Areas of the rat brain drained by the external jugular vein comprise the dorsal and the ventral aspects of the brain excluding the caudal part of the brainstem and the cerebellum which is drained mainly by the vertebral and the small internal jugular vein [29]. The coronal brain segment examined in the present study was limited to the areas drained chiefly by the external jugular vein. This segment comprised the periventricular areas, the major white matter tracts as well as the optic tract and optic chiasma. Multiple sclerosis is a disease of the white matter, the cortical lesions are typically periventricular in $80 \%$ of patients [13].

The present study showed that CCSVI led to iron deposition in the brain, which is consistent with the findings of Zivadinov et al. [53] using MRI in humans. The iron deposits in the present work were recognized mainly in the white matter and the perivascular zones and were detected in oligodendrocytes, a finding similar to the distribution of iron deposits in the brains of more than $30 \%$ of MS patients in a study by Adams [2].

However, the distribution of iron deposits in the present study was different from the normal distribution of iron in the rat brain described by Hill and Switzer [19], in their study iron was mainly localized in the circumventricular organs, the pyriform cortex, the globus pallidus, the caudate-putamen and the substantia nigra. 
Studies showed that chronic venous disease typically leads to iron deposition in tissues [1,48], which is in accordance with the present study.

The iron deposition that was detected in the current study could be explained by reviewing the findings of Sakata et al. [37] who stated that jugular vein ligation alters cerebral venous volume and the findings of Yura et al. [49] that revealed that jugular vein ligation leads to decreased cerebral blood perfusion. Also, decreased CSF reabsorption was linked to bilateral jugular vein ligation [21]. Hypoxic states were shown to facilitate iron uptake [28] and this uptake by the brain is regulated by the blood-brain barrier (BBB) and the blood-cerebrospinal fluid barrier [31]. Such derangement in the CSF flow dynamics might contribute to the iron deposition observed in the present study.

It was suggested that iron initiates the demyelination sequence in MS either directly through the release of free radicals and reactive oxygen [53], or indirectly by acting as a potent chemotactic factor for macrophages and causing the initial activation of T-cell autoimmune cascade [32].

The lesions in MS are characterized by disturbance of the blood-brain barrier, local edema and demyelination; elements which point to an inflammatory process [12]. The inflammatory process in MS is initiated by the expression of adhesion molecules, followed by the passage of T-lymphocytes and macrophages or microglia cells across the blood-brain barrier [23] In the areas of active damage, different populations of lymphocytes dominated by CD8+ T-cells as well as cytotoxic T-cells are found, these cells as well as macrophages and activated microglia are closely attached to degenerating myelin sheaths and axons, the inflammatory cells are believed to play a major role in the demyelination and axonal injury [23].

In spite of the iron deposition detected in the present study, there was no observable demyelination or inflammation in the JVL group which was in agreement with Williams et al. [47] who reported that iron deposits in an animal model of MS were found to be independent of the lesions or the inflammation. These results are contrary to the hypotheses proposed by Zamboni [50] that the deposition of iron as a result of deranged cerebral venous return is capable of inducing signs of MS.

Also in the present study, iron deposits were not seen in macrophages, which is contrary to the findings observed typically in inflammatory diseases [40].
Moreover, it was proposed that the source of perivenular iron deposits in the course of MS is extravasated red blood cells that occur as a consequence to alteration in the blood flow pattern resulting in microbleeds [39]. However, the present study did not reveal the presence of extravasated red blood cells.

The results of the present study were consistent with those rendered by Atkinson et al. [3]. In both studies, bilateral jugular vein ligation resulted in no signs of demyelination with absence of activated microglia and lymphocytes. In both studies, neurological assessment of the rats showed no signs of neurological impairment which was in agreement with the histopathological findings. These findings can explain the existence of CCSVI in up to $30 \%$ of healthy individuals $[22,24]$. These results provide evidence that iron deposition resulting from the venous reflux in CCSVI is not a direct causal factor for inflammatory and demyelinating diseases, which explains its occurrence in disease-free individuals.

The present study showed an increase in the number and size of astrocytes as detected by image analysis of GFAP stained sections, this finding is in accordance with the astrogliosis observed in the course of MS [16]. However, the pattern of astrogliosis in the present study was diffuse while in MS, hypertrophied astrocytes were typically seen inside the acute and chronic lesions and form a limiting zone surrounding chronic and inactive plaques [34].

The occurrence of astrogliosis in the current study could be explained by a decrease in cerebral perfusion [8,37] and decreased regional cerebral oxygen saturation [46]. In another study, Román et al. [36] revealed that gliosis was detected in the brain after incomplete ischemic injuries.

The presence of macrophages and lymphocytes in the active MS lesion was established in several studies $[7,25]$ and immunostaining for CD68 and CD45 to highlight such inflammatory cells in brain tissue was previously reported [10]. However, the present study revealed that both CD68 and CD45 showed no detectable inflammatory response in the JVL group.

In conclusion, there is little convincing evidence to confirm a cause-and-effect relationship between venous insufficiency and the pathophysiological cascade underlying MS using the current animal model of CCSVI. Further studies are recommended in order to assess the possible relation between CCSVI and MS by comparing the effect of induction of CCSVI in 
a chemically-induced rat model of MS, in comparison to an isolated MS model.

\section{Disclosure}

\section{Authors report no conflict of interest.}

\section{References}

1. Ackerman Z, Seidenbaum M, Loewenthal E, Rubinow A. Overload of iron in the skin of patients with varicose ulcers: Possible contributing role of iron accumulation in progression of the disease. Arch Dermatol 1988; 124: 1376-1378.

2. Adams CW. Perivascular iron deposition and other vascular damage in multiple sclerosis. I Neurol Neurosurg Psychiatry 1988; 51 260-265.

3. Atkinson W, Forghani R, Wojtkiewicz GR, Pulli B, Iwamoto Y Ueno T, Waterman P, Truelove J, Oklu R, Chen JW. Ligation of the jugular veins does not result in brain inflammation or demyelination in mice. PLoS One 2012; 7: e33671.

4. Aurboonyawat T, Suthipongchai S, Pereira V, Ozanne A, Lasjaunias P. Patterns of Cranial Venous System from the Comparative Anatomy in Vertebrates Part I, Introduction and the Dorsal Venous System. Interv Neuroradiol 2007; 13: 335-344.

5. Bancroft JD, Gamble M. Theory and practice of histological techniques, $6^{\text {th }}$ ed. Churchill Livingstone, United Kingdom 2008.

6. Bartolomei I, Salvi F, Galeotti R, Salviato E, Alcanterini M, Menegatti E, Mascalchi M, Zamboni P. Hemodynamic patterns of chronic cerebrospinal venous insufficiency in multiple sclerosis: Correlation with symptoms at onset and clinical course. Int Angiol 2010; 29: 183-188.

7. Battistini L, Piccio L, Rossi B, Bach S, Galgani S, Gasperini C, Ottoboni L Ciabini D, Caramia MD, Bernardi G, Laudanna C, Scarpini E, McEver RP, Butcher EC, Borsellino G, Constantin G. CD8+ T cells from patients with acute multiple sclerosis display selective increase of adhesiveness in brain venules: a critical role for P-selectin glycoprotein ligand-1. Blood 2003; 101: 4775-4782.

8. Bousser MG, Chiras J, Bories J, Castaigne P. Cerebral venous thrombosis: a review of 38 cases. Stroke 1985; 16: 199-213.

9. Chen JW, Breckwoldt MO, AikawaE, Chiang G, Weissleder R. Myeloperoxidase-targeted imaging of active inflammatory lesions in murine experimental autoimmune encephalomyelitis. Brain 2008; 131: 1123-1133.

10. Cosenza-Nashat MA, Kim MO, Zhao ML, Suh HS, Lee SC. CD45 Isoform Expression in Microglia and Inflammatory Cells in HIV-I Encephalitis. Brain Pathol 2006; 4: 256-265.

11. Drury R, Wallington E. Carleton's histological techniques. $5^{\text {th }}$ ed. Oxford University Press, England 1980.

12. Hemmer B, Archelos J J, Hartung H P. New concepts in the immunopathogenesis of multiple sclerosis. Nature Rev Neurosci 2002; 3: 291-301.

13. Frohman EM1, Goodin DS, Calabresi PA, Corboy JR, Coyle PK, Filippi M, Frank JA, Galetta SL, Grossman RI, Hawker K, Kachuck NJ, Levin MC, Phillips JT, Racke MK, Rivera VM, Stuart WH. The utility of MRI in suspected MS: report of the Therapeutics and Technology Assessment Subcommittee of the American Academy of Neurology. Neurology 2003; 61: 602-611.
14. Gage GJ, Kipke DR, Shain W. Whole animal perfusion fixation for rodents. J Vis Exp 2012; 65: e3564.

15. Ganesh A, Stahnisch FW. On the historical succession of vessel-based therapies in the treatment of multiple sclerosis. Eur Neurol 2013; 70: 48-58.

16. Genain CP, Cannella B, Hauser SL, Raine CS. Identification of autoantibodies associated with myelin damage in multiple sclerosis. Nat Med 1999; 5: 170-175.

17. Ghahari S, Forwell S J. Social Media Representation of Chronic Cerebrospinal Venous Insufficiency Intervention for Multiple Sclerosis. Int J MS Care 2016; 18: 49-57.

18. Ghezzi A, Annovazzi P, Amato M, Capello E, Cavalla P, Cocco E, Falcini M, Gallo A, Patti F, Perini P, Rodegher ME, Rovaris M, Rottoli MR, Comi G, MS Study Group-Italian Society of Neurology. Adverse events after endovascular treatment of chronic cerebro-spinal venous insufficiency (CCSVI) in patients with multiple sclerosis. Mult Scler 2013; 19: 961-963.

19. Hill JM, Switzer RC. The regional distribution and cellular localization of iron in the rat brain. Neuroscience 1984; 11: 595-603.

20. Khan O, Filippi M, Freedman MS, Barkhof F, Dore-Duffy P, Lassmann H, Trapp B, Bar-Or A, Zak I, Siegel MJ, Lisak R. Chronic cerebrospinal venous insufficiency and multiple sclerosis. Ann Neurol 2010; 67: 286-290.

21. Kotani J, Nitta K, Sakuma Y, Sugioka S, Fujita N, Ueda Y. Effects of bilateral jugular vein ligation on intracranial pressure and cerebrospinal fluid outflow resistance in cats. Br J Oral Maxillofac Surg 1992; 30: 171-173.

22. Lanzillo R, Mancini M, Liuzzi R, Di Donato O, Salvatore E, Maglio V, Vacca G, Amato L, D’Anna G, Brunetti A, Brescia Morra VI. Chronic cerebrospinal venous insufficiency in multiple sclerosis: a highly prevalent age-dependent phenomenon. BMC Neurol 2013; 13: 20

23. Lassmann H. Recent neuropathological findings in MS - implications for diagnosis and therapy Introduction. J Neurol 2004; 251: 2-5.

24. Leone MA, Raymkulova O, Naldi P, Lochner P, Bolamperti L, Coppo L, Stecco A, Liboni W. Chronic cerebrospinal venous insufficiency is not associated with multiple sclerosis and its severity: a blind-verified study. PLoS One 2013; 8: e56031.

25. Lucchinetti C, Brück W, Parisi J, Scheithauer B, Rodriguez M, Lassmann $\mathrm{H}$. Heterogeneity of multiple sclerosis lesions: implications for the pathogenesis of demyelination. Ann Neurol 2000; 47: 707-717.

26. Mandolesi S, Niglio T, Orsini A, De Sio S, d'Alessandro A, Mandolesi D, Fedele F, d'Alessandro A. Venous compression syndrome of internal jugular veins prevalence in patients with multiple sclerosis and chronic cerebro-spinal venous insufficiency. Ann Ital Chir 2016; 87:406-410.

27. Noseworthy JH, Lucchinetti C, Rodriguez M, Weinshenker BG. Multiple sclerosis. N Engl J Med 2000; 343: 938-952.

28. Omori N, Maruyama K, Jin G, Li F, Wang SJ, Hamakawa Y, Sato K, Nagano I, Shoji M, Abe K.Targeting of post-ischemic cerebral endothelium in rat by liposomes bearing polyethylene glycol-coupled transferrin. Neurol Res 2003; 25: 275-279.

29. Paxinos $G$. The rat nervous system, $4^{\text {th }}$ ed. Academic Press, USA 2014 
30. Paxinos G, Watson C. The Rat Brain in Stereotaxic Coordinates. $7^{\text {th }}$ ed. Academic Press, USA 2013.

31. Pinero DJ, Connor JR. Iron and brain function. In: Lieberman HR, Kanarek RB, Prasad C (eds.). Nutritional Neuroscience. CRC Press, Florida 2005; pp. 235-260.

32. Porto G, De Sousa M. Iron overload and immunity. World I Gastroenterol 2007; 13: 4707-4715.

33. Rae-Grant AD, Wong C, Bernatowicz R, Fox RJ. Observations on the brain vasculature in multiple sclerosis: A historical perspective. Mult Scler Relat Disord 2014; 3: 156-162.

34. Raine CS, Scheinberg L, Waltz JM. Multiple sclerosis: oligodendrocyte survival and proliferation in an active established lesion. Lab Invest 1981; 45: 534-546.

35. Reipert B. Multiple sclerosis: a short review of the disease and its differences between men and women. J Men's Health Gen 2004; 1: 334-340.

36. Román GC, Tatemichi TK, Erkinjuntti T, Cummings JL, Masdeu JC, Garcia JH, Amaducci L, Orgogozo JM, Burn A, Hofman A, Moody DM, O’Brien MD, Yamaguchi T, Grafman J, Drayer BP, Bennett DA, Fisher M, Ogata J, Kokmen E, Bermejo F, Wolf PA, Gorelick PB, Bick KL, Pajeau AK, Bell MA, De Carli C, Culebras A, Korczyn AD, Bogousslavsky J, Hartmann A, Scheinberg P. Vascular dementia: diagnostic criteria for research studies. Report of the NINDSAIREN International Workshop. Neurology 1993; 43: 250-260.

37. Sakata K, Endo Y, Kimura F, Yamamoto I. Effects of bilateral jug ular vein ligation on local cerebral blood flow. Skull Base Surg 1999; 9: 207-210.

38. Sengupta P. The Laboratory Rat: Relating Its Age With Human's. Int J Prev Med 2013; 4: 624-630.

39. Sfagos C, Makis AC, Chaidos A, Hatzimichael EC, Dalamaga A Kosma K, Bourantas KL. Serum ferritin, transferrin and soluble transferrin receptor levels in multiple sclerosis patients. Mult Scler 2005; 11: 272-275.

40. Sindrilaru A, Peters T, Wieschalka S, Baican C, Baican A, Peter H, Hainzl A, Schatz S, Qi Y, Schlecht A, Weiss JM, Wlaschek M, Sunderkotter C, Scharffetter-Kochanek K. An unrestrained proinflammatory M1 macrophage population induced by iron impairs wound healing in humans and mice. I Clin Invest 2011; 121: 985-997.

41. Singh AV, Zamboni P. Anomalous venous blood flow and iron deposition in multiple sclerosis. J Cereb Blood Flow Metab 2009; 29: 1867-1878.

42. Sofic E, Paulus W, Jellinger K, Riederer P, Youdim MB. Selective increase of iron in substantia nigra zona compacta of parkinsonian brains. J Neurochem 1991; 56: 978-982.

43. Tsivgoulis G, Faissner S, Voumvourakis K, Katsanos AH, Triantafyllou N, Grigoriadis N, Gold R, Krogias C. "Liberation treatment” for chronic cerebrospinal venous insufficiency in multiple sclerosis: the truth will set you free. Brain Behav 2015; 5: 3-12.

44. Watson C, Paxinos G, Puelles L. The mouse nervous system. $1^{\text {st }}$ ed. Academic Press, USA 2012.

45. Waynforth HB, Flecknell P. Experimental and Surgical Technique in the Rat. $2^{\text {nd }}$ ed. Academic Press, USA 1992.

46. Weir B. Multiple Sclerosis - A Vascular Etiology? Can J Neurol Sci 2010; 37: 745-757.

47. Williams R, Rohr AM, Wang WT, Choi IY, Lee P, Berman NE, Lynch SG, LeVine SM. Iron deposition is independent of cellu- lar inflammation in a cerebral model of multiple sclerosis. BMC Neurosci 2011; 12: 59

48. Yeoh-Ellerton S, Stacey MC. Iron and 8-isoprostane levels in acute and chronic wounds. J Invest Dermatol 2003; 121: 918925.

49. Yura S, Sako K, Yonemasu Y. The effect of disturbance of cerebral venous drainage on focal cerebral blood flow and ischemic cerebral edema. No to Shinkei 1990; 42: 269-275 [abstract].

50. Zamboni P. The Big Idea: Iron-dependent inflammation in venous disease and proposed parallels in multiple sclerosis. J R Soc Med 2006; 99: 589-593.

51. Zamboni P, Consorti G, Galeotti R, Gianesini S, Menegatti E, Tacconi G, Carinci F. Venous Collateral Circulation of the Extracranial Cerebrospinal Outflow Routes. Curr Neurovasc Res 2009; 6: 204-212.

52. Zamboni P, Galeotti R, Menegatti E, Malagoni AM, Tacconi G, Dall'Ara S, Bartolomei I, Salvi F. Chronic cerebrospinal venous insufficiency in patients with multiple sclerosis. J Neurol Neurosurg Psychiatry 2009; 80: 392-399.

53. Zivadinov R, Schirda C, Dwyer MG, Haacke ME, Weinstock-Guttman B, Menegatti E, Heininen-Brown M, Magnano C, Malagoni AM, Wack DS, Hojnacki D, Kennedy C, Carl E, Bergsland N, Hussein S, Poloni G, Bartolomei I, Salvi F, Zamboni P. Chronic cerebrospinal venous insufficiency and iron deposition on susceptibility-weighted imaging in patients with multiple sclerosis: a pilot case-control study. Int Angiol 2010; 29: 158-175. 